\title{
Remember your driver
}

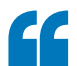

the promoters

of SWI5 and

CLB2 might

recruit factors

co-transcriptionally

to accompany

transcripts into

the cytoplasm
The stability of mRNA is often assumed to be dictated by a transcript's sequence features. Two new studies highlight that mRNA stability can be influenced by a memory of the promoter from which expression of the transcript was driven.

Spurred on by their previous discovery that components of the transcription apparatus mediate cytoplasmic mRNA degradation, Bregman et al. tested the effect of different promoters on mRNA stability by using reporter constructs to express the usually unstable RPL30 transcript in Saccharomyces cerevisiae. After chemically blocking transcription, the kinetics of mRNA degradation were monitored using northern blotting. When the RPL30 upstream activating sequence (UAS) was exchanged for the UAS of the ACT1 gene, the stability of the same $R P L 30$ transcript was increased to a level that was similar to that of the endogenous ACT1 transcript, indicating that the promoter sequence was a key determinant of stability.

To identify which sequences were mediating the effect, the authors dissected the UASs to make new constructs. They found that
Rap1-binding sites (RapBSs) in the RPL30 UAS were necessary for $R P L 30$ transcript instability and were sufficient to confer transcript instability when engineered into the ACT1 UAS. Depletion of Rap1 caused transcripts to stabilize, particularly those from constructs with RapBSs in the UAS. Therefore, promoterinduced mRNA degradation may involve Rap1 binding to RapBSs and a co-transcriptional 'imprinting' of that transcript for cytoplasmic degradation, although mechanistic details are unclear.

In a related study, Trcek et al. developed an RNA-fluorescence in situ hybridization (RNA-FISH) technique to study mRNA stability at single-molecule resolution in unperturbed S. cerevisiae. They studied two transcripts, SWI5 and $C L B 2$, for which transcription and degradation are closely regulated during the cell cycle. Exchanging the 5' and $3^{\prime}$ untranslated regions (UTRs) of the SWI5 transcript with UTRs from constitutively expressed ACT1 did not perturb the M-phase-specific degradation of SWI5. Instead, only promoter swapping with ACT1 converted unstable, cell-cycle-regulated
SWI5 and CLB2 transcripts into stable, constitutively expressed transcripts and vice versa.

The authors reasoned that the promoters of SWI5 and CLB2 might recruit factors co-transcriptionally to accompany transcripts into the cytoplasm to regulate $\mathrm{M}$-phase-specific transcript degradation. Protein interaction databases identified Dbf2 as a candidate for a role in this process based on it being at the intersection of relevant pathways. Indeed, Dbf2 was bound to SWI5 and CLB2 mRNAs in vivo, and depletion of Dbf2 destabilized these mRNAs and delayed $M$ phase progression. This indicates that Dbf 2 accompanies and protects specific transcripts until appropriate cell-cycle-regulated degradation signals are received.

It will be interesting to uncover precisely how the promoter-initiated events lead to the selective induction or prevention of cytoplasmic mRNA degradation. Finally, because other yeast promoters share sequences with RPL30, SWI5 and CLB2, it will be intriguing to decipher how widespread and varied promoterregulated mRNA stability is throughout yeast and metazoan genomes.

Darren J. Burgess

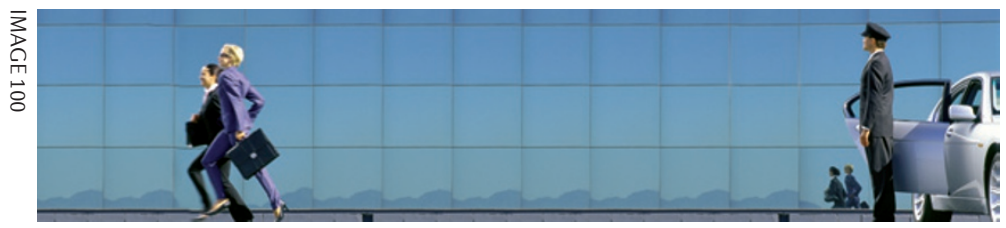

ORIGINAL RESEARCH PAPERS Bregman, A. et al. Promoter elements regulate cytoplasmic mRNA decay. Cell 147, 1473-1483 (2011) | Trcek, T. et al. Single-molecule mRNA decay measurements reveal promoter-regulated mRNA stability in yeast. Cell 147, 1484-1497 (2011) 